\title{
Peritoneal Fluid Cytology of Disseminated Large Cell Neuroendocrine Carcinoma Combined with Endometrioid Adenocarcinoma of the Endometrium
}

\author{
Yong-Moon Lee, Min-Kyung Yeo ${ }^{1}$, Song-Yi Choi ${ }^{1}$, Kyung-Hee Kim ${ }^{1}$, Kwang-Sun Suh ${ }^{1}$ \\ Department of Pathology, Dankook University School of Medicine, Cheonan; 'Department of Pathology, Chungnam National University School of Medicine, Daejeon, Korea
}

\begin{abstract}
Primary large cell neuroendocrine carcinoma (LCNEC) of the endometrium is extremely rare ${ }^{1-3}$ and the cytomorphology has not been well described. Recently, we experienced a case of combined LCNEC with endometrioid carcinoma (ECa) showing peritoneal dissemination that was confirmed by histology and peritoneal fluid cytology. The purpose of this report is to delineate cytologic characteristics of LCNEC in an effusion specimen.
\end{abstract}

\section{CASE REPORT}

A 62-year-old woman was admitted for evaluation of continuous vaginal bleeding for 1 month. She was in a menopausal state since the age of 50 years with gravida 2-para 2, and had no specific remarkable past medical history. Her initial laboratory test was unremarkable. Ascites was noticed on physical examination. On ultrasonography, the uterine corpus was enlarged with the endometrium thickened to $15 \mathrm{~mm}$. Endometrial curettage showed low-grade ECa. Intraoperative peritoneal fluid sampling demonstrated small-sized tumor cell clusters measuring approximately $100-150 \mu \mathrm{m}$ in diameter and discohesive polyhedral single tumor cells admixed with karyorrhectic debris, which made a definitive diagnosis difficult. In contrast to mesothelial cells and lymphocytes in the background, tumor cells had large nuclei with an irregular nuclear membrane, vesicular nuclei, relatively prominent nucleoli, and notable cytoplasm (Fig. 1A-C). A panel of

Received: June 11, 2019 Revised: July 19, 2019

Accepted: July 29, 2019

Corresponding Author: Kwang-Sun Suh, MD, PhD

Department of Pathology, Chungnam National University School of Medicine,

266 Munhwa-ro, Jung-gu, Daejeon 35015, Korea

Tel: +82-42-280-7196, Fax: +82-42-280-7189, E-mail: kssuh@cnu.ac.kr immunohistochemical stains was performed on the cell block material, and atypical cells were positive for CD56 (Fig. 1D) and synaptophysin, but not for chromogranin and CD45. These findings were consistent with neuroendocrine carcinoma (NEC). An en-bloc resection was performed. On microscopic examination, there were foci of transition between a low-grade ECa and a loosely cohesive carcinoma component in the endometrium (Fig. 2A) invading the superficial myometrium (Fig. 2B). The loosely cohesive tumor component showed pseudoglandular and cord-like growth patterns. The tumor cells had relatively abundant cytoplasm, vesicular nuclei, and prominent nucleoli (Fig. 2C). There were tumor emboli in lymphovascular spaces of the myometrium. These cells were positive for CD56 (Fig. 2D) and synaptophysin (Fig. 2E), but negative for CD45 and CD99. Some tumor cells were positive for epithelial membrane antigen (Fig. 2F) and cytokeratin. According to these findings, a diagnosis of combined LCNEC with a low-grade ECa was made. The patient died of the disease 32 days after operation.

\section{Ethics statement}

Approval for this case report was obtained from the Institutional Review Board (IRB) of Chungnam National University Hospital (CNUH-IRB 2017-9-49) with a waiver of informed consent.

\section{DISCUSSION}

LCNEC is characterized by the presence of polygonal cells with a neuroendocrine growth pattern in at least part of the tumor and expression of one or more of the neuroendocrine markers (chromogranin, CD56, and synaptophysin) in more than $10 \%$ 

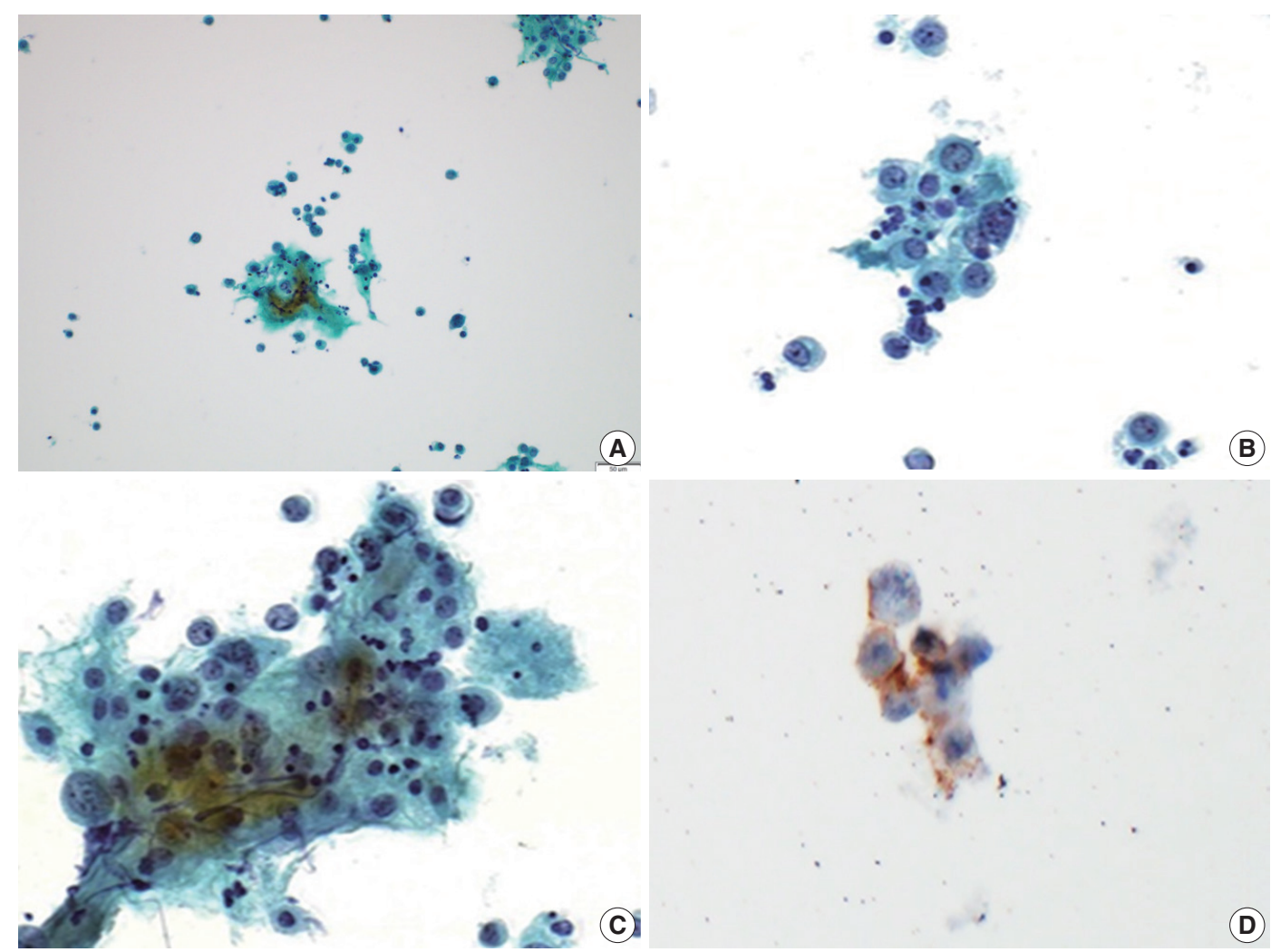

Fig. 1. Cytologic features of large cell neuroendocrine carcinoma in a peritoneal fluid smear. (A) Loose clusters of tumor cells measuring 100 to $150 \mu \mathrm{m}$ are present. $(\mathrm{B}, \mathrm{C})$ These tumor cells are polyhedral with abundant eosinophilic cytoplasm, nuclei are either vesicular or hyperchromatic, chromatin is heterogeneous, and nucleoli are variably prominent. (D) These cells show positive reactions for CD56.

of tumor cells. ${ }^{1}$ Due to the rarity of LCNECs in the female genital tract, ${ }^{1-3}$ a specific diagnosis of LCNEC is usually not possible in effusion specimens. Additional difficulties that may be encountered in effusion cytology include overlapping morphology among similar neoplastic entities, scant cellularity, and predominance of apoptosis or cellular debris. Cytopathologic diagnosis of LCNEC is more challenging than small cell neuroendocrine carcinoma due to rare nuclear molding (13\%), frequent apoptosis (67\%), and prominent nucleoli (86\%) in LCNEC. ${ }^{4}$ The cytologic findings of LCNEC of the uterine cervix are characterized by loosely cohesive clusters or single tumor cells with hyperchromatic nuclei and necrotic materials in the background. The ovoid nuclei are 3-5 times larger than nuclei of small lymphocytes and have coarsely clumped chromatin and two or more prominent nucleoli. The tumor cells have a moderate amount of cytoplasm. ${ }^{5-7}$

A cytopathologic differential diagnosis of our case included serous carcinoma, small cell NEC (SCNEC), and undifferentiated carcinoma. Serous carcinoma is characterized by marked exfoliation of high-grade tumor cells either in the form of cellular clusters or single cells. Psammoma bodies can be seen. ${ }^{8}$ SCNEC is com- posed of relatively uniform small hyperchromatic nuclei with characteristic nuclear molding and scant cyanophilic cytoplasm. ${ }^{9}$ In this case, cellular overlapping, an acinar arrangement, and papillary configuration were not distinctive. Dedifferentiated carcinoma is composed of a mixture of undifferentiated carcinoma and either the International Federation of Gynecology and Obstetrics grade 1 or $2 \mathrm{ECa} .{ }^{1}$ Undifferentiated carcinoma grows as sheets of noncohesive atypical tumor cells without any nested or trabecular architecture and displays chromogranin and/or synaptophysin staining in a minority of tumor cells. ${ }^{10}$ In this case, the tumor components showed a cord-like growth pattern and were positive for $\mathrm{CD} 56$ and synaptophysin.

In high-grade NEC metastases, the three architectural patterns of the cytomorphologic spectrum that have been presented within body cavities are (1) a predominance of small clusters of tumor cells (seen more often in LCNEC cases), (2) a predominance of large clusters of tumor cells (mainly in SCNEC), and (3) a predominance of single tumor cells (seen in both SCNECs and LCNECs). ${ }^{4}$ The small clustering pattern is seen in $73 \%$ of LCNEC and $41 \%$ of SCNEC cases. ${ }^{4}$ Our case presented predominantly with small clusters of tumor cells measuring less 

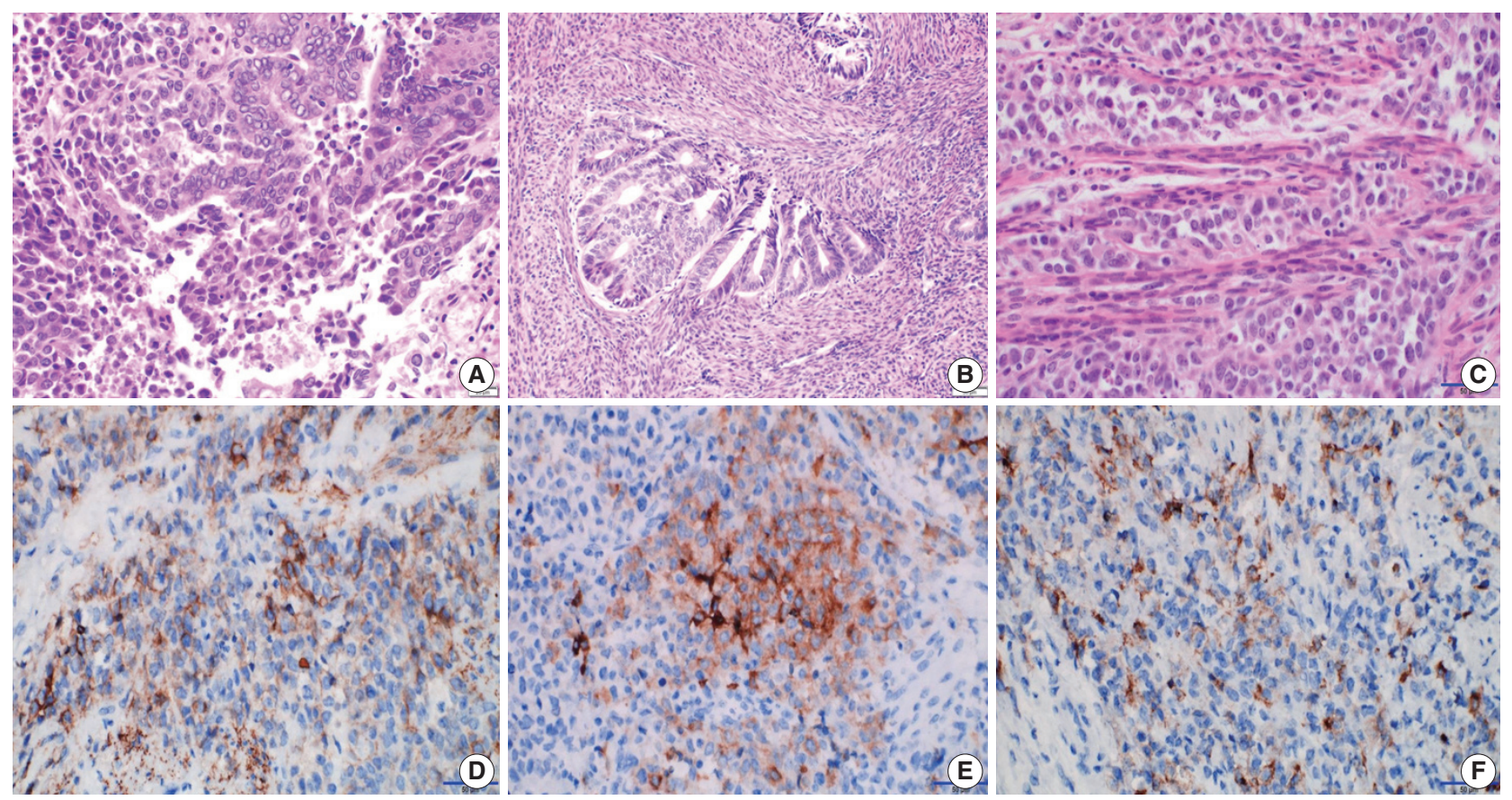

Fig. 2. Microscopic and immunohistochemical findings. The endometrium shows foci of transition between a low-grade endometrioid adenocarcinoma and a loosely cohesive carcinoma component (A) with a cribriform pattern of growth invading the myometrium (B). (C) The loosely cohesive tumor component shows a cord-like growth pattern and the tumor cells have relatively abundant eosinophilic cytoplasm with a large polyhedral nucleus and prominent nucleoli. Immunohistochemically, these tumor cells show positive reactions for CD56 (D) and synaptophysin (E). (F) Some tumor cells are positive for epithelial membrane antigen.

than $150 \mu \mathrm{m}$ (approximately 100 to $150 \mu \mathrm{m}$ ) and discohesive single tumor cells.

\section{ORCID}

Yong-Moon Lee: https://orcid.org/0000-0002-4302-9263

Min-Kyung Yeo: https://orcid.org/0000-0001-8873-0021

Song-Yi Choi: https://orcid.org/0000-0002-8496-5613

Kyung-Hee Kim: https://orcid.org/0000-0003-0214-0296

Kwang-Sun Suh: https://orcid.org/0000-0003-3874-864X

\section{Author Contributions}

Conceptualization: KHK, KSS.

Data curation: YML, MKY.

Funding acquisition: KSS.

Writing—original draft: YML, KSS.

Writing—review \& editing: MKY, SYC, KSS.

\section{Conflicts of Interest}

The authors declare that they have no potential conflicts of interest.

\section{Funding}

This study was supported by the research fund of Chungnam National University.

\section{REFERENCES}

1. Kurman RJ, Carcangiu ML, Herrington CS, Young RH. WHO classification of tumours of female reproductive organs. 4th ed. Lyon: IARC Press, 2014; 131-3.

2. Chun YK. Neuroendocrine tumors of the female reproductive tract: a literature review. J Pathol Transl Med 2015; 49: 450-61.

3. Deodhar KK, Kerkar RA, Suryawanshi P, Menon H, Menon S. Large cell neuroendocrine carcinoma of the endometrium: an extremely uncommon diagnosis, but worth the efforts. J Cancer Res Ther 2011; 7: 211-3.

4. Khalbuss WE, Yang H, Lian Q, Elhosseiny A, Pantanowitz L, Monaco $\mathrm{SE}$. The cytomorphologic spectrum of small-cell carcinoma and large-cell neuroendocrine carcinoma in body cavity effusions: a study of 68 cases. Cytojournal 2011; 8: 18.

5. Lee WY. Exfoliative cytology of large cell neuroendocrine carcinoma of the uterine cervix. Acta Cytol 2002; 46: 1176-9.

6. Niwa K, Nonaka-Shibata M, Satoh E, Hirose Y. Cervical large cell 
neuroendocrine carcinoma with cytologic presentation: a case report. Acta Cytol 2010; 54(5 Suppl): 977-80.

7. Kuroda N, Wada Y, Inoue K, et al. Smear cytology findings of large cell neuroendocrine carcinoma of the uterine cervix. Diagn Cytopathol 2013; 41: 636-9.

8. Kobayashi TK, Norimatsu Y, Buccoliero AM. Cytology of the body of the uterus. In: Gray W, Kocjan G, eds. Diagnostic cytopathology. 3rd ed. London: Churchill Livingstone Elsevier, 2010; 709-11.

9. Tabbara SO, Khalbuss WE. Other malignant neoplasms. In: Nayar $\mathrm{R}$, Wilbur DC, eds. The Bethesda system for reporting cervical cytology. 3rd ed. Cham: Springer, 2015; 244-59.

10. Altrabulsi B, Malpica A, Deavers MT, Bodurka DC, Broaddus R, Silva EG. Undifferentiated carcinoma of the endometrium. Am J Surg Pathol 2005; 29: 1316-21. 